Meteorology and Atmospheric Physics manuscript No.

(will be inserted by the editor)

\title{
Detrended fluctuation analysis of daily temperature records: Geographic dependence over Australia
}

\author{
Andrea Király, Imre M. Jánosi \\ Department of Physics of Complex Systems, Eötvös University, Pázmány Péter sétány 1, H-1117 Budapest, Hungary. \\ Tel:+(36)-(1)-372-2878, fax:+(36)-(1)-372-2866, E-mail: janosi@lecso.elte.hu
}

Received: date / Revised version: date

\begin{abstract}
Daily temperature anomaly records are analyzed (61 for Australia, 18 for Hungary) by means of detrended fluctuation analysis. Positive long range asymptotic correlations extending up to 5-10 years are detected for each case. Contrary to earlier claims, the correlation exponent is not universal for continental stations. Interestingly, the dominant factor is geographic latitude over Australia: the general tendency is a decrease of correlation exponent with increasing distance from the equator. This tendency is in a complete agreement with the results found by Tsonis et al. (1999) for 500-hPa height anomalies in the northern hemisphere. The variance of fluctuations exhibits an opposite trend, the larger is the distance from the equator, the larger the amplitude of intrinsic fluctuations. The presence of Tropospheric Biennial Oscillation is clearly identified for three stations at the north-eastern edge of the Australian continent.
\end{abstract}

Key words Daily temperature anomalies, Scaling, Tropospheric Biennial Oscillation

\section{Introduction}

Sophisticated detection studies demonstrate that the mean global surface temperature has increased by $\sim 0.06^{\circ} \mathrm{C}$ per decade in the 20 th Century and by $0.19^{\circ} \mathrm{C}$ per decade between 1978 and 1998 (Houghton et al., 2001). All projections of future change indicate that the warming is likely to continue, and this conclusion holds regardless of the computer model used or the emission scenario applied in the model (Zwiers, 2002). Besides an improving reproduction of current mean atmospheric state, confidence in the simulation and prediction skills of global climate models requires the reproduction of long-time correlation properties revealed by measurements. A recent test by Govindan et al. (2002) of seven state-ofthe-art global models failed to reproduce the scaling behavior of six long temperature records by underestimat- ing the long range persistence of the atmosphere. Similar discrepancies were detected already by Syroka and Toumi (2001a, 2001b). Quite the contrary, Fraedrich and Blender (2003) demonstrated that long range correlations can be reproduced by a numerical model if the coupling with ocean is represented properly (although with fixed composition of greenhouse gases). In any case, direct comparison of local observations with the rather low resolution general circulation models might have many pitfalls (Pielke et al., 2002), therefore an extended analysis of measured asymptotic correlations is a prerequisite.

Various methods are used to characterize quantitatively the fluctuations and correlations of high frequency meteorological data. Power density spectra (Marple 1987; Percival and Walden 1993) have been routinely computed for decades. Pelletier (1997) determined the temperature spectra for hundreds of stations and ice core records and identified power-law behavior for continental and maritime locations. Koscielny-Bunde et al. (1998) reported to observe a near universal exponent value in the fluctuations of daily temperatures, however Weber and Talkner (2001) found differences depending on the altitude of the meteorological station. Probably the largest related study up to now is presented by Eichner et al. (2003) on temperature records of 95 stations all over the globe. They obtained correlation exponent values in the range 0.55-0.9 centered strongly at around 0.65 over continents, but systematically higher values for islands. The last observation is supported by Monetti et al. (2003), where they detected much stronger persistence for sea surface temperature than values for air over ground. Tsonis et al. (1999) demonstrated scale invariance in the variability of $500-\mathrm{hPa}$ height anomalies and found a general tendency for the correlation exponent to decrease with increasing latitude. In general, scaling can be identified asymptotically from the longest available records only, shorter-time correlations are almost fully explained by using first or second order linear autoregressive models (von Storch and Zwiers 1999; Király and Jánosi 2002). 
In this work we exploit the method of detrended fluctuation analysis (DFA) which has proven useful in revealing the extent of long range correlations in diverse time series (Peng et al. 1994, 1995). Talkner and Weber (2000), and Heneghan and McDarby (2000) pointed out that DFA and traditional power spectra provide equivalent characterizations of correlated stochastic signals, with the essential difference that DFA can effectively filter out slow trends. The situation is similar to the connection of power spectra and autocorrelation functions manifested by the Wiener-Khintchin theorem: the information content is mathematically the same, but in many cases spectral density functions are more sensitive and better exploratory tools for real data (von Storch and Zwiers 1999).

Our analysis is based on a high-quality daily temperature data set for Australia (Torok and Nicholls 1996; Trewin and Trevitt 1996). The same collection was utilized in many studies to reveal an increased wheat yield due to recent climate trends (Nicholls 1997), changing maximum and minimum temperature trends for the globe (Easterling et al. 1997), Australian land surface temperature variability (Jones 1999), trends in extreme daily rainfall and temperature in Southeast Asia (Manton et al. 2001), the impact of land cover change on the Australian near-surface climate (Narisma and Pitman 2003), etc. Data for 61 (out from 107) stations were selected according to the quality of their climate record, in terms of site standards, homogeneity and completeness of the series, and to provide the best possible spatial coverage (48 for the continent, 13 for islands). The average time span of the records was 45 years with a minimum of 22 and a maximum of 120 years. We evaluated also historical records of daily mean temperatures measured at 18 weather-stations in Hungary from 1951 until 1989. Amplitude distributions, power spectra and autocorrelation functions were analyzed earlier essentially for the same data set by Jánosi and Vattay (1992), asymptotic scaling and stochastic modeling have been described by Király and Jánosi (2002).

Since the DFA method is relatively new, we provide its concise exposition in Section 2. Section 3 gives the details of our results, Section 4 is devoted to a short discussion of the relevance and consequences of the findings.

\section{Detrended fluctuation analysis}

The most important advantage of DFA over conventional methods (e.g., autocorrelation-, spectral- and Hurst-analysis) is that it permits the detection of intrinsic selfsimilarity embedded in a seemingly nonstationary time series. Following the work of Peng et al. (1994, 1995), several theoretical studies elucidated the power and limitations of filtering out various trends from synthetic data series (Heneghan and McDarby 2000; Talkner and We- ber 2000; Hu et al. 2001; Kantelhardt et al. 2001; Chen et al. 2002).

We consider a fluctuating time series $x_{i},(i=1, \ldots, N)$ sampled at equidistant times $i \Delta t$. We assume that $x_{i}$ are increments of a random walk process around the average $\langle x\rangle=N^{-1} \sum_{i=1}^{N} x_{i}=0$, thus the "trajectory" or "profile" of the signal is given by integration as

$$
y_{j}=\sum_{i=1}^{j} x_{i} .
$$

We divide the profile into nonoverlapping segments of equal length $n$ indexed by $k=1, \ldots,[N / n]$. In each segment, the local trend is fitted by a polynomial $f_{k}^{(p)}(j)$ of order- $p$ [see Fig. [1(a)], and the profile is detrended by subtracting this local fit: $z_{j}^{(p)}=y_{j}-f_{k}^{(p)}(j), j=1, \ldots, N$. A possible measure of fluctuations can be given by the root mean square

$$
F_{p}(n)=\sqrt{\frac{1}{n[N / n]} \sum_{j=1}^{n[N / n]}\left(z_{j}^{(p)}\right)^{2}}
$$

for a given segment length $n$. A power-law relationship between $F_{p}(n)$ and $n$ indicates scaling with an exponent $\delta$ (DFA $p$ exponent):

$$
F_{p}(n) \sim n^{\delta}
$$

Notice that such a process has a power-law autocorrelation function

$$
C(\tau)=\left\langle x_{j} x_{j+\tau}\right\rangle \sim \tau^{-\alpha},
$$

where $0<\alpha<1$, and the relationship between the correlation exponents is (Koscielny-Bunde et al. 1998; Talkner and Weber 2000)

$$
\alpha=2(1-\delta)
$$

Consequently, long-memory (persistent) processes are characterized by a DFA exponent $\delta>0.5$, uncorrelated time series (e.g., pure random walk) obey $\delta=0.5$, antipersistent signals exhibiting negative long range correlations have $\delta<0.5$. (Here the terms "persistent" and "antipersistent" are used in the sense that an increasing trend in the past implies an increasing or decreasing trend in the future, thus it slightly differs from "persistence" in climatology defined as the continuance of a specific pattern.)

Fig. 1(b) shows typical results for a particular station (Gunnedah). As a first step of the analysis, the annual cycle is removed from the raw data $T_{i}$ (daily mean temperatures) by computing the temperature anomaly series

$$
x_{i}=T_{i}-\left\langle T_{i}\right\rangle_{d},
$$

where \langle\rangle$_{d}$ denotes the long-time average value for the given calendar day. The curves in Fig. 1(b) represent the results for DFA1, DFA2 and DFA3 evaluation, where 
local trends were removed by linear, second-, and thirdorder polynomials. The low noise level is due to the standard "sliding window" technique, where local trend removal and variance computation for a given time window $n$ were performed at each possible starting value $i=1, \ldots, N-n$. The shapes are practically the same indicating that there is no significant trend in the temperature anomaly series (DFA $p$ would filter out an overall polynomial trend of order $p-1$ resulting in changing asymptotic slopes). Note that this observation does not contradict recent studies on global warming for the same geographic area, because relatively weak trends are easily masked by the variability of daily average temperatures, thus careful statistical analyses of extremes are proven more convincing (Easterling et al. 1997; Manton et al. 2001; Frich et al. 2002). The gradual downward shift (decreased overall variance for a given segment length $n$ ) is a simple consequence of that local trends are better approximated by polynomials of higher order [c.f. Fig. 1(a)]. For short times $(n<20$ day), the curves exhibit a gradually decreasing slope to a well defined asymptotic value $\delta=0.75 \pm 0.01$ for the particular case, the statistics breaks down for very large segment lengths $(n>2000$ day $\approx$ one fifths of the total length).

Systematic analyses by Hu et al. (2001), Kantelhardt et al. (2001) and Chen et al. (2002) with various synthetic time series revealed many useful details on the efficiency of the method. One of their main findings is that DFA results for signals with different correlation properties and background trends can be fully explained by the assumption of variance superposition. This is essential because the correlation exponent is almost never constant for any real data, crossover(s) can usually arise from a change in the correlation properties at different time scales or as a consequence of trends. Other types of nonstationarities are missing segments in records (very common), contamination with random spikes, or signals with different local behavior (different variance or local correlations). From our point of view, the most important cases are the following (Hu et al. 2001; Kantelhardt et al. 2001; Chen et al. 2002):

1. Long range correlated process with additive random noise. The typical DFA curve has a crossover from a slope $\delta=0.5$ (noise dominated part) to a different asymptotic value. The crossover time depends primarily on the variance of the random noise (large amplitude - later crossover) and on the correlation exponent of the underlying process (large exponent earlier crossover).

2. Noise with sinusoidal trend. A pure sinusoidal signal has a DFA $p$ curve of initial slope $\delta=p+1$ with a crossover to $\delta=0$. (Obviously, the variance is limited by the total amplitude in time windows larger than the half period of the harmonic signal.) Together with noise, the resulting DFA $p$ curve is a superposition of both ingredients.
3. Signals with segments removed. Surprisingly, scaling of correlated data series $(\delta>0.5)$ is not affected by randomly cutting out segments and stitching together the remaining parts, even when $50 \%$ of the points are removed (Chen et al. 2002). Nevertheless the applicability of this result for temperature records with long missing segments is limited, because the removal of the annual cycle can be problematic.

4. Signals with different local correlations. In general, when random parts of a correlated data series is replaced by segments from another series of different correlation exponent, the behavior is dominated by the segments exhibiting higher positive correlations (Chen et al. 2002). However, there is a wide transition regime with a nontrivial exponent, thus a "true" asymptotic behavior could be observed at very long time series only, especially when the difference between the exponents is small. We will return to the relevance of this point in Section 4 (Discussion).

Finally, we emphasize that we observed weak overall trends in the longest temperature anomaly series (Sydney, Melbourne, Adelaide) which might be attributed to urbanization or global warming, but this question is beyond the scope of the present work. Even for these cases, DFA2 or higher order exponents could not be distinguished from DFA1 values within the fitting error.

\section{Results}

We analyzed 61 daily maximum, minimum and mean temperature series for Australian stations, and 18 daily mean temperature records for Hungary. We found asymptotic long range correlations in the range 30-1800 days for each case, the fitting regime of power-law behavior extends up to 10 years for the longest records. In strong contrast to earlier claims on a universal exponent value (Koscielny-Bunde et al. 1998; Govindan et al. 2002), we found pronounced station dependence.

\subsection{Correlation exponent}

Figure2 illustrates how the value of correlation exponent $\delta$ is connected with the geographic location of the station over Australia. Here DFA analysis was performed for 48 daily mean temperature anomaly series, the behavior of daily minima and maxima is practically the same. Two tendencies can be resolved in Fig. 2 Firstly, the general trend is a decrease of exponents with decreasing latitude. Indeed, the contours of the fitted surface are almost parallel with the lines of latitude. This tendency is in complete agreement found by Tsonis et. al. (1999) for $500-\mathrm{hPa}$ height anomalies in the northern hemisphere, which indicates that the key factor is the distance from the equator. Secondly, there is a hump over the southeastern part of the continent which correlates well with 
the location of the highest mountain range (Australian Alps). We do not want to overemphasize this observation, nevertheless Weber and Talkner (2001) found also higher exponent values for mountain weather stations.

The decreasing tendency over the Australian continent is clear also in Fig. 3 where the same exponent $\delta$ is plotted as a function of latitude for every analyzed stations. Error bars are obtained from fits to different segments for the scaling regime of DFA curves. A comparison with Hungarian data (stars in Fig. 3) indicate another aspect of the the lack of universality: geographic latitude is not a dominant factor over the Carpathian Basin. Note that a full symmetry of the northern and southern hemispheres is not expected, also the differences of climates should be reflected in differing correlation properties. As for the islands, Weber and Talkner (2001), and Monetti et. al. (2003) already reported on systematically higher level of correlations for maritime stations and sea surface temperatures, which is in agreement with our results (see Fig. 31). It is interesting that the trend for islands is also decreasing upto $50^{\circ} \mathrm{S}$. The number of cases below this latitude is not enough to conclude whether the trend is reversed there, or what we see is just localized anomaly. These four stations (in latitudinal order: Macquarie Island, Casey, Mawson, and Davis) belong essentially to Antarctic climate.

It is remarkable that our main result (decreasing exponents with increasing distance from the equator) is not visible on the map given by Fraedrich and Blender (2003), which might be a consequence of their much lower spatial resolution over Australia.

In order to reveal another possible determining factors, we show in Fig. 4 scatter plots for the exponent value $\delta$ and different geographic parameters. Hungarian data in Fig. 目 (stars) indicate that longitude is a dominating factor over this region, unfortunately the narrow spatial coverage restricts an unambiguous conclusion. This behavior is reflected also in Fig. 迎, but this is an obvious consequence of geographic locations: whatever is the reference point (Mediterranean Sea or Atlantic Ocean), the distance from the shore is increasing as the longitude increases.

As for Australia, Fig. 目 can be explained by accepting that latitude is the determining factor, and there is no correlation with longitude. In this case we expect a distribution reflecting somehow the shape of the continent. Indeed, the higher density of stations at the southwest corner is seemed to be projected in Fig. 四. Cumulated exponent values show weak correlation with the elevation, see Fig. 4b. Note that this does not contradict to the interpretation we gave for the ridge in Fig. 2 because this scatter plot smears differences in longitude and latitude of stations. As for the distance from the oceans (Fig. 固), the dependence is week again. High exponent values can be associated with islands, but there is no significant drop as we move away from the shore. This does not mean that the distance from large water masses can not contribute to the degree of correlation, but the apparent effect is weak over Australia.

Fig. 四 shows the scatter plot for $\delta$ and the DFA variance at a fixed temporal window size, but let us return to this point after describing the general properties of variance itself, in the next Subsection.

\subsection{Variance}

In order to characterize the average variance of fluctuations, we plotted $F_{1}(n=108)$ (see Eq. 2) for daily mean temperature anomalies in Fig. [5 as a function of geographic location. Daily minima and maxima show the same behavior (but not necessarily the same numerical value), again. The particular time lag might seem to be somewhat arbitrary, however any other choice in the range $100<n<500$ resulted in the same statistics, apart from numerical values. Too large time lags are not suitable, because the DFA curves for different stations have different slopes, and crossing confounds the relationship between stations. The general trend for variance over the continent is not surprising: the proximity of ocean damps temperature fluctuations, which is a well known and well documented fact.

Figure 6] shows the variance statistics for every stations as a function of geographic latitude. There is a trend of increasing variance with increasing distance from the equator, especially for the islands. This tendency can be revealed also by marking continental stations on the shore (solid circles with crosses in Fig. [6), however the dispersion is rather large. Variances far from the oceans are systematically increased both for Australia and Hungary (Fig. 6).

The correlation plot between average variance and DFA exponents is shown in Fig. 41. Based on Fig. 3 3 and Fig. [6] we expect that stations far away from the equator obey a relatively low exponent value but large variance, this negative correlation is clearly visible in Fig. $4 \mathrm{~d}$. The scatter for continental stations is pretty large, thus oneto-one coupling remains hidden (if it exists at all) by other modifying factors, such as the distance from ocean or elevation.

\subsection{Detection of Tropospheric Biennial Oscillation}

In a few cases, DFA curves exhibited characteristic kinks shown in Fig. 77(a), which became more pronounced at higher order local detrending. This behavior is consistent with the presence of a periodic sinusoidal background trend (Hu et al. 2001). Unfortunately, there is no way to decompose such an underlying component, especially because it is far from being pure harmonic indicated by the shift of breakpoints [see Fig. [7(a)].

The existence of a slow, smeared oscillation was actually clear for three stations, namely Cairns $\left(16.88^{\circ} \mathrm{S}\right.$, 
$\left.145.27^{\circ} \mathrm{E}\right)$, Thursday Island $\left(10.58^{\circ} \mathrm{S}, 142.21^{\circ} \mathrm{E}\right)$, and Willis Island $\left(16.30^{\circ} \mathrm{S}, 149.98^{\circ} \mathrm{E}\right)$; a further time series (Darwin, $12.42^{\circ} \mathrm{S}, 130.88^{\circ} \mathrm{E}$ ) indicated a weak signal of similar behavior. For these cases, even the standard autocorrelation analysis [Fig. 7(b)] revealed statistically significant periodicity of $1.9 \pm 0.2$ year (note that this is an average considering also higher harmonics). The very stations are located at the north-eastern edge of the continent (Fig. 3), and they exhibit the lowest intrinsic variance, see Fig. 5] [Actually, the lowermost variance is found at Cocos (Keeling) Island $\left(12.07^{\circ} \mathrm{S}, 96.83^{\circ} \mathrm{E}\right)$, c.f. Fig. 6] but it is situated far to the west in the Indian Ocean.) No other stations have shown statistically significant periodic components.

The location of these places suggests that the observed periodic trend might be connected with the wellknown El-Niño Southern Oscillation (ENSO), that is the quasi-periodic disruption of the Walker circulation forced by a pressure gradient over the Pacific. The changes in pressure distribution are measured by the Southern Oscillation Index (SOI), which is far from being regular with a period of 2-7 years (Ropelewski and Jones 1987; Allan et. al. 1991). A direct comparison of the autocorrelation functions for the daily mean temperature anomalies at Willis Island [Fig. [7(b)] and for the SOI signal [Fig. 7(c)] shows immediately that the two oscillations are not strongly coupled.

Biennial signals in rainfall, sea-level pressure, seasurface temperature and other climate elements in the tropical Indian and western Pacific regions have been known and studied for years (e.g., Trenberth 1975; Meehl $1987 ; 1997)$. The phenomenon is termed as Tropospheric Biennial Oscillation (TBO). This TBO has an irregular period of 2-3 years and shows eastward movement. Several theories and hypotheses have been proposed, however the exact mechanism is not clearly known. A strong biennial component is seen in the ENSO phenomena (Ropelewski et. al. 1992) and it is accepted to be an integral part of the Asia-Pacific climate. Since the slow oscillation observed for the stations above has a characteristic period of two years [see Fig. 7(b)], we can consider it as a fingerprint of TBO. As far as we know, no other daily surface data series of similar length have shown such markedly the presence of two-year oscillation.

\section{Discussion}

Our main findings can be summarized as follows.

(i) Daily temperature values have significant positive long range correlations extending for several years. There is no sign of breakdown of scaling behavior even for the longest time series.

(ii) The "degree" of correlations, i.e., the value of correlation exponent is not universal, it depends on the geographic location. (iii) There is a general tendency of decreasing exponent value with increasing distance from the equator over Australia.

(iv) The variance of fluctuations has an opposite trend: the larger the distance from the equator, the larger the intrinsic variance.

Let us comment shortly points (ii) and (iii). As for the lack of universality, (ii), an opposite conclusion could be drawn from DFA analysis of a few time series only (Koscielny-Bunde et al. 1998; Govindan et al. 2002). Similar tests by Weber and Talkner (2001), Monetti et. al. (2002), Fraedrich and Blender (2003), and especially Eichner et al. (2003) do indicate significant differences as a function of climatic parameters of the stations. Different exponent values for different places do not exclude the existence of a universal mechanism behind the long memory effects, such as coupling with the oceans. Nevertheless any speculation on such a mechanism should be preceded by further analyses of asymptotic correlations with the highest possible spatial resolution.

The decreasing tendency of correlation exponents over Australia is in complete agreement with the results by Tsonis et. al. (1999) for 500-hPa height anomalies in the northern hemisphere. Their fluctuation analysis is essentially identical with a DFA1 method, even exponent values can be compared. They attribute a decreasing degree of correlations to an increasingly baroclinic nature of the dynamics as one progresses from the subtropics through the midlatitudes. More baroclinicity results in more power to processes of small spatial and temporal scales. Our results support this picture in the following way. Whatever is the source of a decreased correlation exponent at higher latitudes, it can not be decomposed to a core dynamics and an additive random noise. This is because the DFA method very effectively identifies such a process. Tuning of the exponent value is possible, e.g., by replacing random parts of a correlated signal with segments from another series of different correlation properties (see Section 1). In this respect, a dynamics of increased baroclinicity means that an underlying correlated process can be more often interrupted by short-memory, small scale processes yielding a decreased effective correlation exponent.

\section{Acknowledgements}

We would like to thank Tamás Tél for useful recommendations and for his permanent encouragement. This work was supported by the Hungarian Science Foundation (OTKA) under Grant Nos. T032423 and T032437.

\section{References}

1. Allan, R. J., Nicholls, N., Jones, P. D. and Butterworth, I. J. (1991) A further extension of the Tahiti-Darwin 
SOI, Early ENSO events and Darwin pressure. J. Climate, 4: 743-749.

2. Caballero, R., Jewson, S. and Brix, A. (2002) Long memory in surface air temperature: detection, modeling, and application to weather derivative valuation. Clim. Res., 21: 127-140.

3. Chen, Z., Ivanov, P. C., Hu, K. and Stanley, H. E. (2002) Effect of nonstationarities on detrended fluctuation analysis. Phys. Rev. E, 65: 041107.

4. Easterling, D. R., et al. (1997) Maximum and minimum temperature trends for the globe. Science, 277: 364-367.

5. Eichner, J. F., et al. (2003) Power-law persistence and trends in the atmosphere: A detailed study of long temperature records. Phys. Rev. E, 68: 046133.

6. Fraedrich, K. and Blender, R. (2003) Scaling of atmosphere and ocean temperature correlations in observations and climate models. Phys. Rev. Lett., 90: 108501.

7. Frich, P. et al. (2002) Observed coherent changes in climatic extremes during the second half of the twentieth century. Clim. Res., 19: 193-212.

8. Govindan, R. B., Vyushin, D., Bunde, A., Brenner, S., Havlin, S. and Schellnhuber, H. J. (2002) Global climate models violate scaling of the observed atmospheric variability. Phys. Rev. Lett., 89: 028501.

9. Heneghan, C. and McDarby, G. (2000) Establishing the relation between detrended fluctuation analysis and power spectral density analysis for stochastic processes. Phys. Rev. E, 62: 6103-6110.

10. Houghton, J. T., Ding, Y., Griggs, D. J., Noguer, M., van der Linden, P. J., Dai, X.,Maskell, K. and Johnson, C. A., eds. (2001) Climate Change 2001: The Scientific Basis. Cambridge University Press, Cambridge.

11. Hu, K., Ivanov, P. C., Chen, Z., Carpena, P. and Stanley, H. E. (2001) Effect of trends on detrended fluctuation analysis. Phys. Rev. E, 64: 011114.

12. Jánosi, I. M. and Vattay, G. (1992) Soft turbulent state of the atmospheric boundary layer. Phys. Rev. A, 46: 63866389 .

13. Jones, D. A. (1999) Characteristics of Australian land surface temperature variability. Theor. Appl. Climat., 63: 11-31.

14. Kantelhardt, J. W., Koscielny-Bunde, E., Rego, H. H. A., Havlin, S. and Bunde, A. (2001) Detecting long-range correlations with detrended fluctuation analysis. Physica A,295: 441-454.

15. Király, A. and Jánosi, I. M. (2002) Stochastic modeling of daily temperature fluctuations. Phys. Rev. E,65: 051102.

16. Koscielny-Bunde, E., Bunde, A., Havlin, S., Roman, H. E., Goldreich, Y. and Schellnhuber, H.-J. (1998) Indication of universal persistence law governing atmospheric variability. Phys. Rev. Lett., 81: 729-732.

17. Manton, M. J. et al. (2001) Trends in extreme daily rainfall and temperature in Southeast Asia and the South Pacific: 1961-1998. Int. J. Climatol., 21: 269-284.

18. Marple, S. L. (1987) Digital Spectral Analysis with Applications. Prentice-Hall, New Jersey.

19. Meehl, G. A. (1987) The annual cycle and interannual variability in the tropical Pacific and Indian Ocean region. Mon. Wea. Rev., 115: 27-50.

20. Meehl, G. A. (1997) The South Asian monsoon and the Tropospheric Biennial Oscillation. J. Climate, 10: 19211943.
21. Monetti, R. A., Havlin, S. and Bunde, A. (2003) Long term persistence in the sea surface temperature fluctuations. Physica A, 320: 581-589.

22. Narisma, G. T. and Pitman, A. J. (2003) The impact of 200 years of land cover change on the Australian nearsurface climate. J. Hydrometeorol, 4: 424-436.

23. Nicholls, N. (1997) Increased Australian wheat yield due to recent climate trends. Nature, 387: 484-485.

24. Pelletier, J. D. (1997) Analysis and modeling of the natural variability of climate. J. Climate, 10: 1331-1342.

25. Peng, C.-K., Buldyrev, S. V., Havlin, S., Simons, M., Stanley, H. E. and Goldberger, A. L. (1994) Mosaic organization of DNA nucleotides. Phys. Rev. E., 49: 1685-1689.

26. Peng, C.-K., Havlin, S., Stanley, H. E. and Goldberger, A. L. (1995) Quantification of scaling exponents and crossover phenomena in nonstationary heartbeat time series Chaos, 5: 82-87.

27. Percival, D. B. and Walden, A. T. (1993) Spectral Analysis for Physical Applications. Cambridge University Press, Cambridge.

28. Pielke, R. A., Stohlgren, T., Schell, L., Parton, W., Doesken, N., Redmond, K., Moeny, J., McKee, T. and Kittel, T. G.F. (2002) Problems in evaluating regional and local trends in temperature: An example from eastern Colorado. Int.l J. Climat., 22: 421-434.

29. Ropelewski, C. F. and Jones, P. D. (1987) An extension of the Tahiti-Darwin Southern Oscillation Index. Mon. Wea. Rev., 115: 2161-2165.

30. Ropelewski, D. F., Halpert, M. S. and Wang, X. (1992) Observed tropospheric biennial variability and its relationship to the Southern Oscillation. J. Climate, 5: 594-614.

31. von Storch, H. and Zwiers, F. W. (1999) Statistical Analysis in Climate Research. Cambridge University Press, Cambridge.

32. Syroka J. and Toumi R. (2001a) Scaling of Central England temperature fluctuations? Atmos. Sci. Lett., 2: 143154.

33. Syroka J. and Toumi R. (2001b) Scaling and persistence in observed and modeled surface temperature. Geophys. Res. Lett., 28: 3255-3258.

34. Talkner, P. and Weber, R. O. (2000) Power spectrum and detrended fluctuation analysis: application to daily temperatures. Phys. Rev. E, 62: 150-160.

35. Torok, S. J. and Nicholls, N. (1996) A historical annual temperature data set for Australia. Aust. Met. Mag., 45: 251-260.

36. Trenberth, K. E. (1975) A quasi-biennial standing wave in the Southern Hemisphere and interrelations with sea surface temperature. Q. J. R. Meteorol. Soc., 101: 55-74.

37. Trewin, B. C. and Trevitt, A. C. F. (1996) The development of composite temperature records. Int. J. Climat., 16: $1227-1242$.

38. Tsonis, A. A., Roebber, P. J. and Elsner, J. B. (1999) Long-range correlations in the extratropical atmospheric circulation: Origin and implications. J. Climate, 12: 15341541.

39. Weber, R. O. and Talkner, P. (2001) Spectra and correlations of climate data from days to decades. J. Geophys. Res., 106: 20131-20144.

40. Zwiers, F. W. (2002) Climate change: The 20-year forecast. Nature, 416: 690-691. 

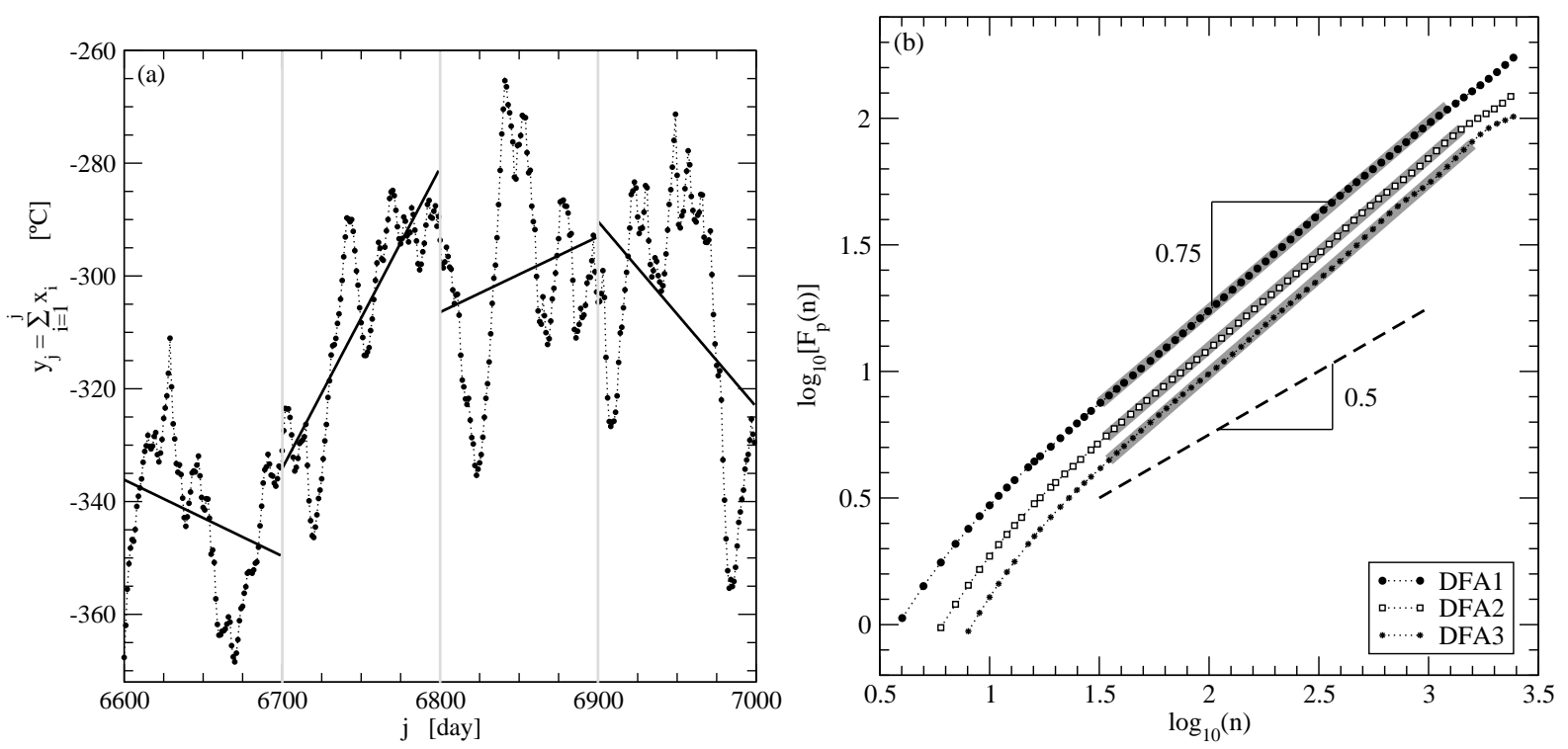

Fig. 1 (a) Local linear fits (solid line) for an integrated temperature anomaly series $y_{j}$ [see (1)] divided into segments of length $n=100$. (b) DFA results for a daily mean anomaly series (Gunnedah 1969-1995, 31.02 ${ }^{\circ}$ south, $150.27^{\circ}$ east). Gray lines indicate scaling with an exponent $\delta=0.75 \pm 0.01$, dashed line illustrates the slope for an uncorrelated process.

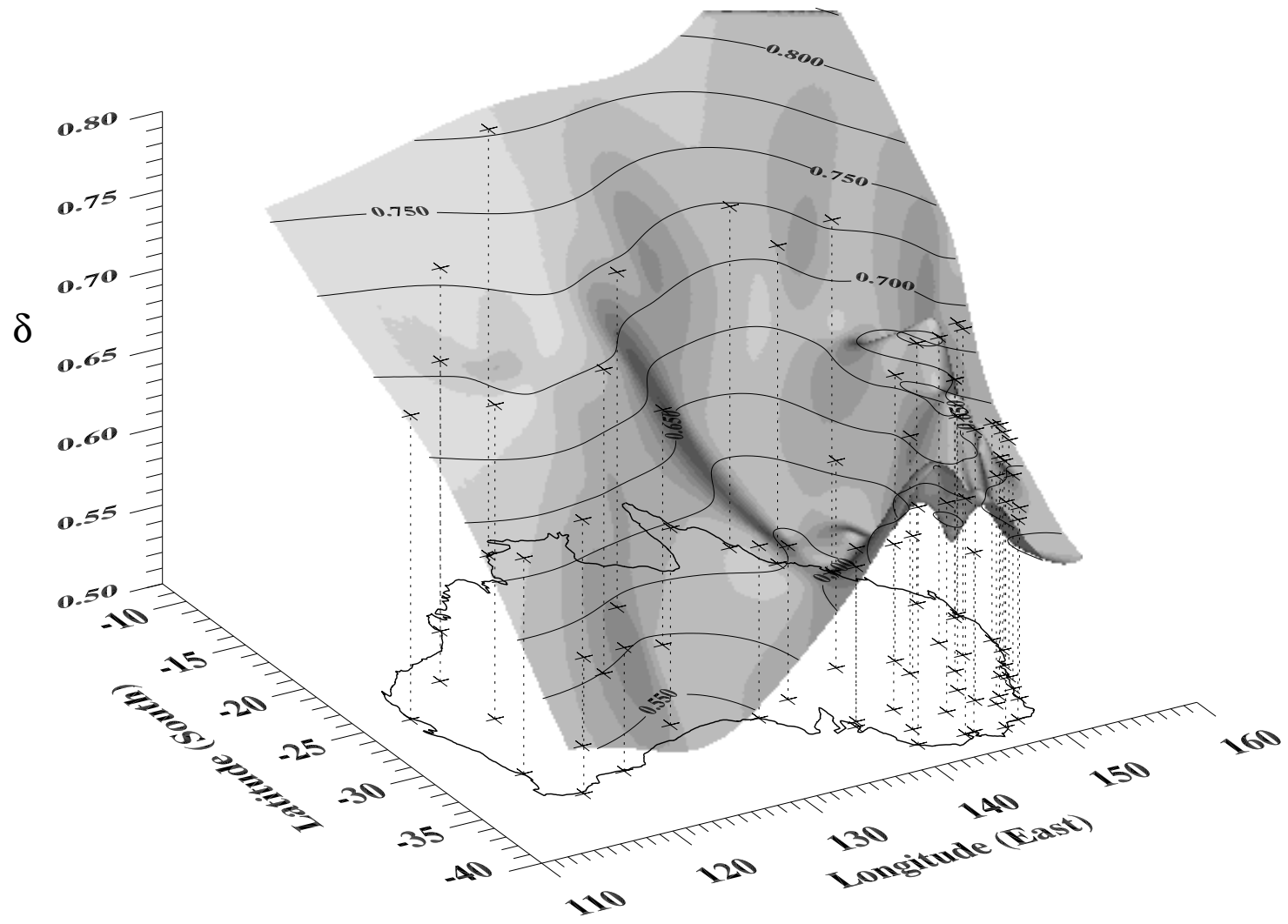

Fig. 2 Correlation exponent $\delta$ for daily mean temperature anomalies at the continental stations (48 altogether) as a function of geographic location. The contour of Australia is also indicated. (Error bars are shown in Fig. 3) 

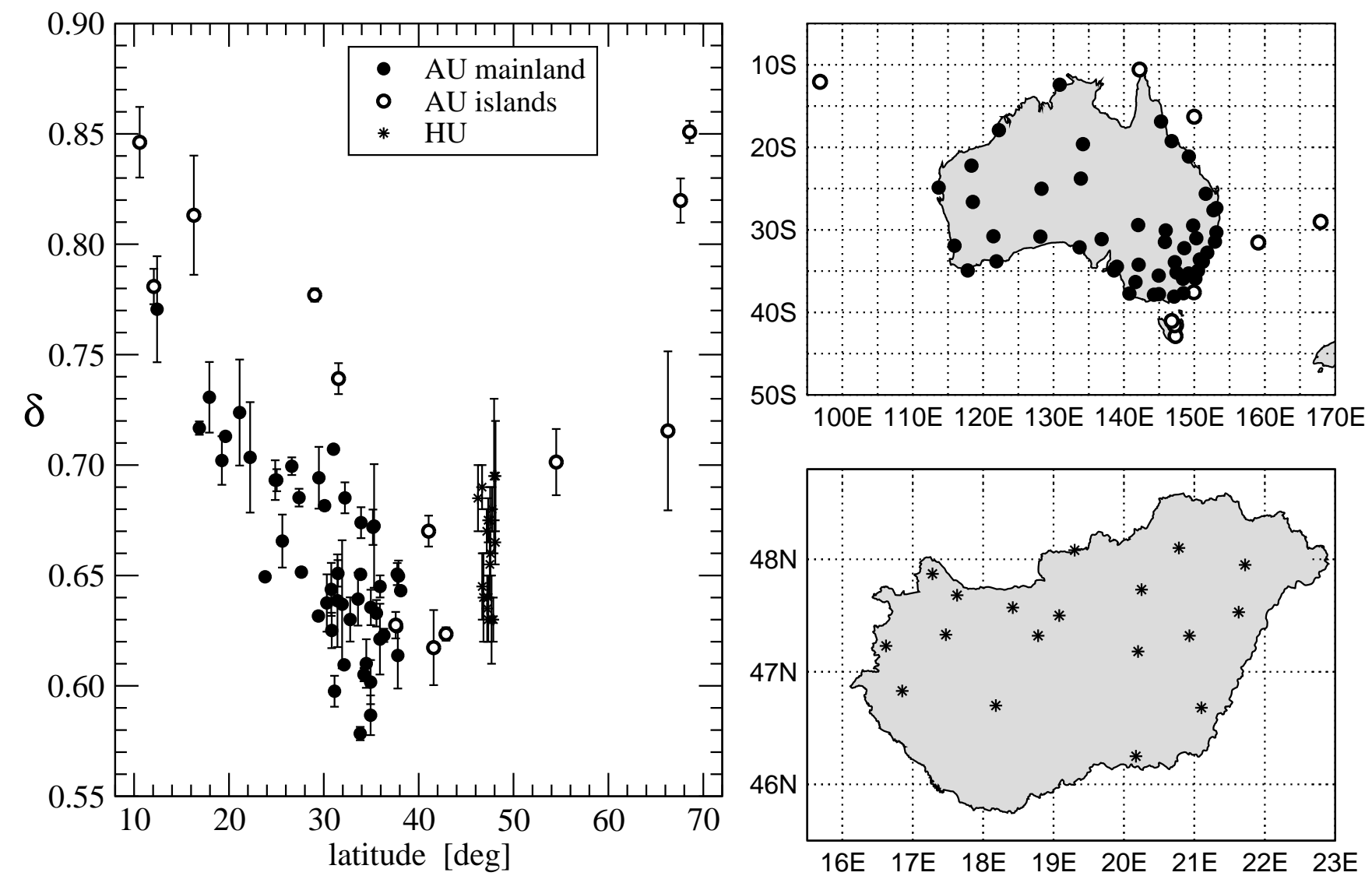

Fig. 3 Correlation exponent $\delta$ for daily mean temperature anomalies as a function of geographic latitude. Heavy dots: Australian continent; empty circles: islands (note that locations south from the line $50^{\circ} \mathrm{S}$ are not indicated in the map); stars: Hungarian stations. The legend box does not hide data. 

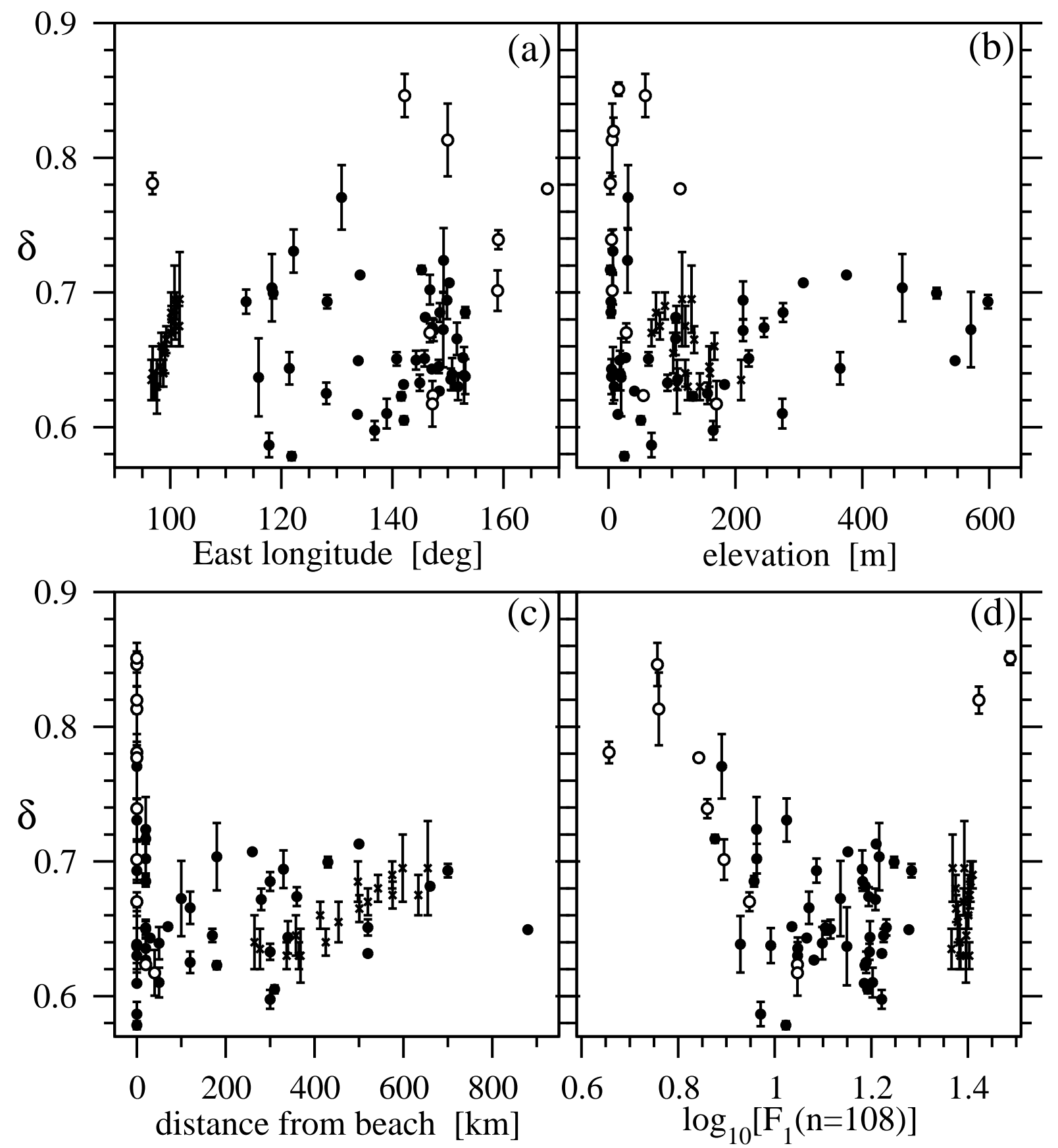

Fig. 4 Scatter plot for the correlation exponent $\delta$, and (a) the longitude (values for Hungary are shifted by $80^{\circ}$ ), $(\mathbf{b})$ the elevation, (c) the distance from the closest seashore (Trieste for Hungarian stations), and (d) the logarithm of average variance of fluctuations for time window $n=108$ days. Heavy dots: Australian mainland; empty circles: islands; stars: Hungarian stations. 

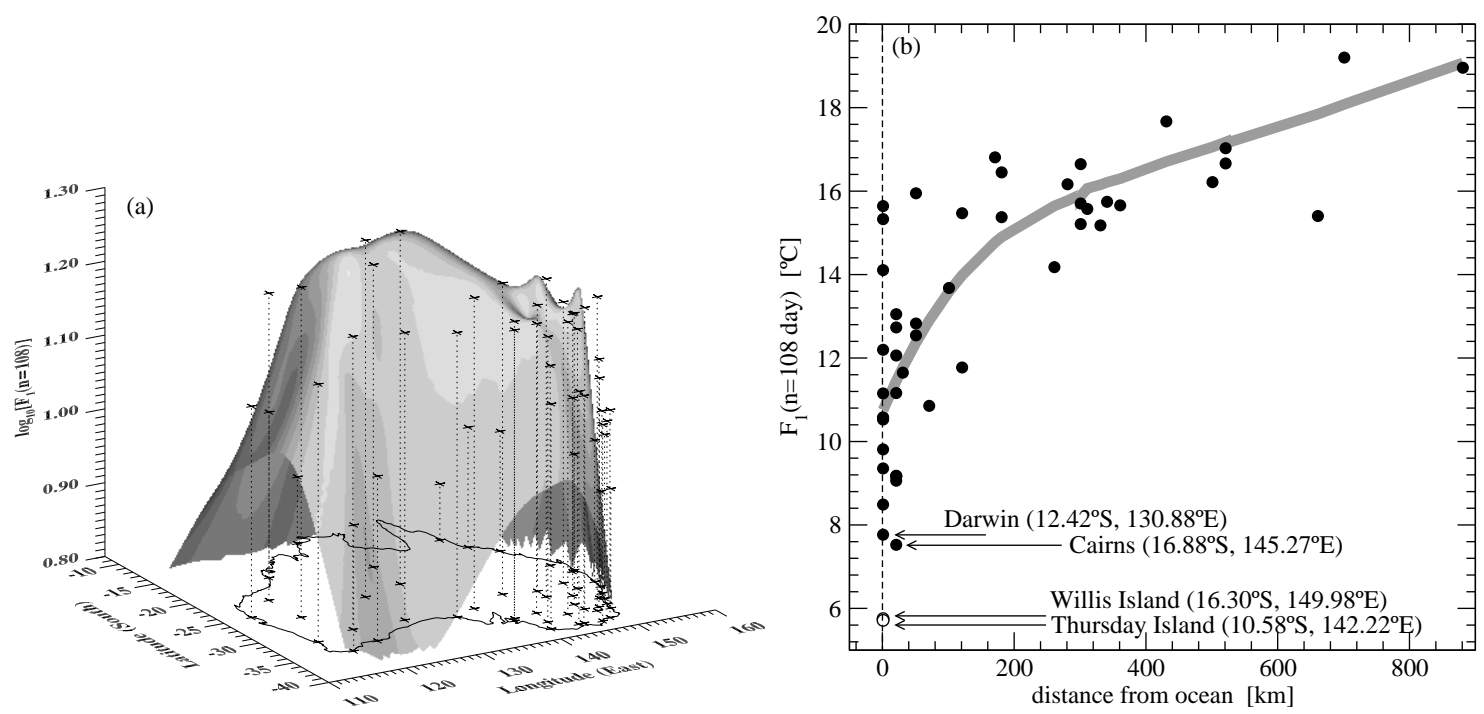

Fig. 5 Average variance of fluctuations for daily mean temperature anomalies for time window $n=108$ day. $\left(\right.$ a): $\log _{10}\left[F_{1}(n=\right.$ 108)] (see Eq. 2) at the location of stations on the Australian mainland. (b): $F_{1}(n=108)$ as a function of distance from the ocean. (The gray line only guides the eye.) Stations of smallest variance are identified [see Section 3(c)].

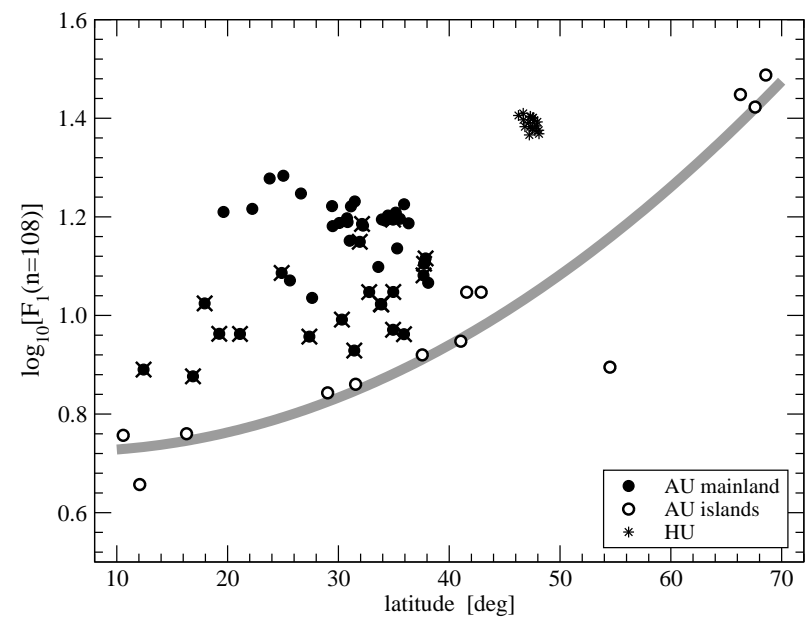

Fig. 6 Logarithm of average variance of fluctuations for daily mean temperature anomalies $F_{1}$ for time window $n=108$. Solid circles: Australian mainland; solid circles with crosses: Australian shore (distance from the ocean is less than $20 \mathrm{~km}$ ); empty circles: islands; stars: Hungarian stations. The gray line only guides the eye. 

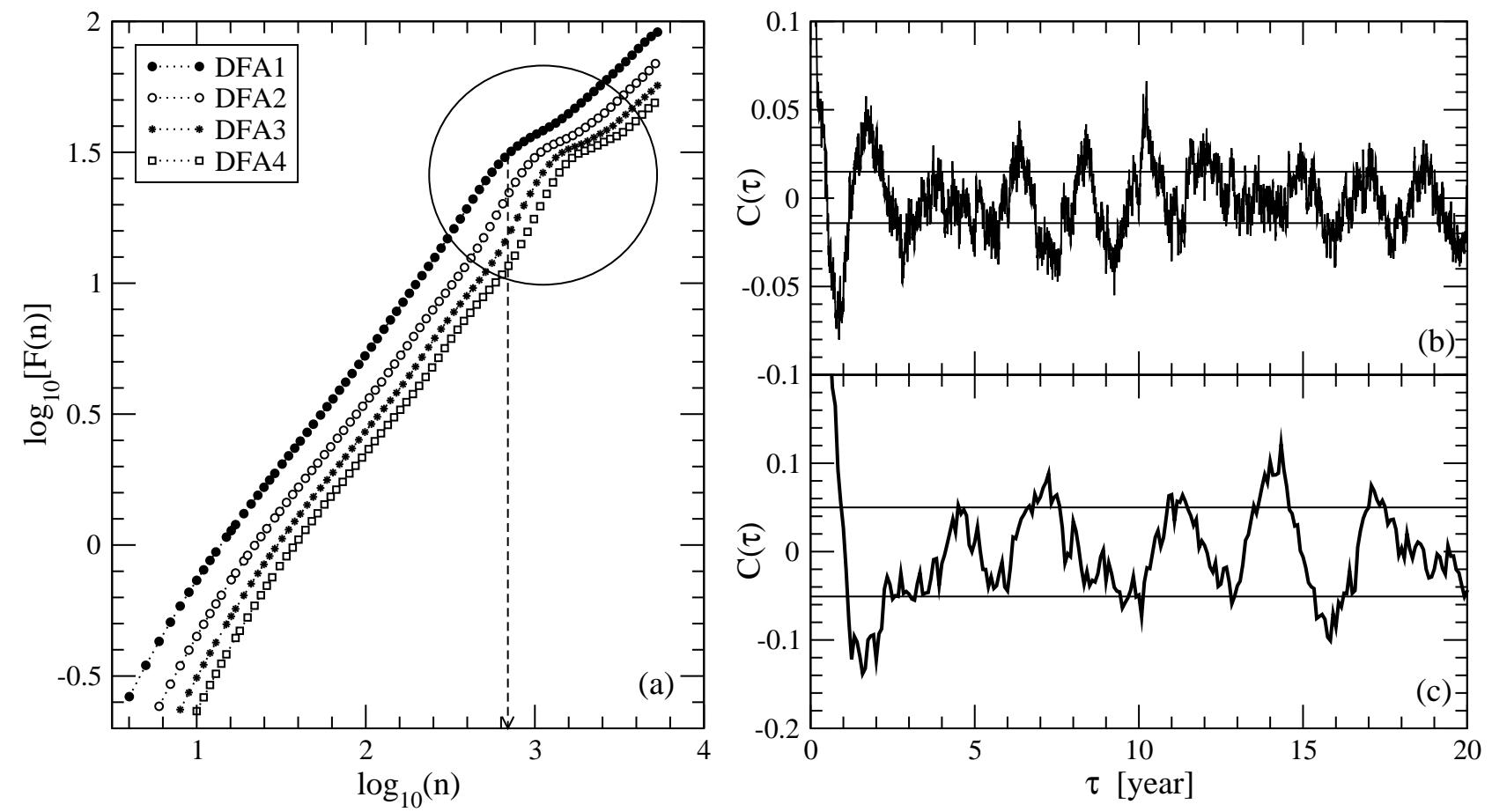

Fig. 7 (a) DFA analysis for Willis Island $\left(1939-1999,16.30^{\circ} \mathrm{S}, 149.98^{\circ} \mathrm{E}\right)$. Circle indicates the kink in the curves characteristic for a quasi-periodic background cycle of $\sim 1.9$ years (vertical dashed line). (b) Autocorrelation function for the same series (solid line), the lag $\tau$ in units of years. (c) Autocorrelation for the Southern Oscillation Index (1866-1995) from the University of East Anglia (ftp://daac.gsfc.nasa.gov/data/inter_disc/surf_temp_press/soi), $\tau$ in units of years. Thin lines indicate $95 \%$ confidence level for both autocorrelation functions. 